\title{
The mineral composition and spatial distribution of the dust ejecta of NGC $6302^{\star, \star \star}$
}

\author{
F. Kemper ${ }^{1}$, F. J. Molster ${ }^{2}$, C. Jäger ${ }^{3}$, and L. B. F. M. Waters ${ }^{1,4}$ \\ 1 Astronomical Institute “Anton Pannekoek”, University of Amsterdam, Kruislaan 403, 1098 SJ Amsterdam, The Netherlands \\ 2 ESTEC/ESA, RSSD-ST, Keplerlaan 1, 2201 AZ Noordwijk, The Netherlands \\ 3 University of Jena, Astrophysical Institute and University Observatory (AIU), Schillergässchen 3, 07745 Jena, Germany \\ ${ }^{4}$ Instituut voor Sterrenkunde, K.U. Leuven, Celestijnenlaan 200B, 3001 Heverlee, Belgium
}

Received 22 May 2002 / Accepted 30 July 2002

\begin{abstract}
We have analysed the full ISO spectrum of the planetary nebula NGC 6302 in order to derive the mineralogical composition of the dust in the nebula. We use an optically thin dust model in combination with laboratory measurements of cosmic dust analogues. We find two main temperature components at about 100 and $50 \mathrm{~K}$ respectively, with distinctly different dust compositions. The warm component contains an important contribution from dust without strong infrared resonances. In particular the presence of small warm amorphous silicate grains can be excluded. The detection of weak PAH bands also points to a peculiar chemical composition of the dust in this oxygen-rich nebula. The cool dust component contains the bulk of the mass and shows strong emission from crystalline silicates, which contain about 10 percent of the mass. In addition, we identify the $92 \mu \mathrm{m}$ band with the mineral calcite, and argue that the $60 \mu \mathrm{m}$ band contains a contribution from the carbonate dolomite. We present the mass absorption coefficients of six different carbonate minerals. The geometry of the dust shell around NGC 6302 is studied with mid-infrared images obtained with TIMMI2. We argue that the cool dust component is present in a circumstellar dust torus, while the diffuse emission from the warm component originates from the lobes.
\end{abstract}

Key words. planetary nebulae: individual: NGC 6302 - stars: circumstellar matter - dust, extinction - methods: laboratory

\section{Introduction}

All low and intermediate mass stars end their life on the Asymptotic Giant Branch (AGB) by ejecting their entire Hrich envelope through a slowly expanding, dusty wind. After the AGB, the central star quickly increases its effective temperature to values high enough to begin ionizing its AGB ejecta: a planetary nebula (PN) is born. Depending on the mass and thus luminosity of the star, the transition from AGB to PN can go very fast: for the most massive objects, in less than 1000 years ionization of the nebula begins. These massive objects therefore are characterized by dense AGB remnants and a very hot luminous central star.

NGC 6302 is probably one of the best studied PNe with a massive progenitor. A recent determination of the mass of the ionized nebula is about $2 M_{\odot}$ (Pottasch \& Beintema 1999),

Send offprint requests to: F. Kemper,

e-mail: ciska@science.uva.nl

* Based on observations with ISO, an ESA project with instruments funded by ESA Member States (especially the PI countries: France, Germany, The Netherlands and the UK) and with the participation of ISAS and NASA.

$\star \star$ Based on observations made with ESO Telescopes at the La Silla or Paranal Observatories under programme ID 67.D-0132(A). based on a distance determination of $1.6 \mathrm{kpc}$ (Gómez et al. 1993). Dust mass estimates also indicate a very massive shell $\left(M_{\mathrm{d}}=0.02 M_{\odot}\right)$, using a distance of $2.2 \mathrm{kpc}$ (Gómez et al. 1989). These distance determinations rely on VLA observations of the expansion of the nebula over a $2.75 \mathrm{yr}$ period, assuming an outflow velocity of $13 \mathrm{~km} \mathrm{~s}^{-1}$. In principle, this is a very reliable method, however the epoch over which the nebula is observed, is very short. Therefore the increase in size is very small and hard to measure, indicating that the error bars on these distance determinations are still very large. Instead, we adopt a distance of $0.91 \mathrm{kpc}$, based on emission-line photometry of NGC 6302, from which the $B$ and $V$ magnitudes, the luminosity and the distance can be derived (Shaw \& Kaler 1989). The large number of luminous PNe studied gives confidence in the distance determination from this statistical method.

The nebular abundances indicate that NGC 6302 is a type I nebula, consistent with a massive progenitor. Casassus et al. (2000) estimate that the progenitor mass is $4-5 M_{\odot}$. The morphology of the nebula observed at optical wavelengths is highly bipolar, pointing to non-spherical mass loss on the AGB, resulting in a dusty torus in the equatorial region (Lester \& Dinerstein 1984). The inclination angle of the system is $\sim 45^{\circ}$ with respect to the line-of-sight (Bohigas 1994). The temperature of the central star is very high: Casassus et al. (2000) 
mention a temperature of $\sim 250000 \mathrm{~K}$, while Pottasch et al. (1996) arrive a temperature of $\sim 380000 \mathrm{~K}$. Although there is some uncertainty about the distance and therefore masses and luminosities involved, everything points to a rather massive and luminous progenitor.

The Infrared Space Observatory (ISO) spectrum has been presented in several papers (Barlow 1998; Molster et al. 2001a, $2002 \mathrm{~b}$ ) and is characterized by a wealth of narrow solid state features in the $20-70 \mu \mathrm{m}$ spectral range caused by circumstellar dust in the AGB remnant, as well as by strong emission lines from a multitude of fine-structure lines originating from the ionized gas in the nebula. The dust bands have been identified with crystalline silicates and a number of other components (Koike et al. 2000; Molster et al. 2001a, 2002a). Kemper et al. (2002b, hereafter referred to as Paper I) have reported on the detection of carbonates in the dust shell, based on the identification of broad features at $\sim 60$ and $\sim 92 \mu \mathrm{m}$.

Optical and near-infrared images have already shown that the dust distribution around NGC 6302 is rather complex (Lester \& Dinerstein 1984; Schwarz et al. 1992). The ISO spectroscopy supports this, because a broad dust temperature range is required to explain the shape of the spectral energy distribution. In addition the dust composition seems to be very complex, as there is evidence for a mixed chemistry by the presence of both oxygen-rich dust and carbon-rich dust features, the latter in the form Polycyclic Aromatic Hydrocarbons (PAHs) (see Molster et al. (2001a) for a discussion on the origin of this dichotomy).

In order to reconstruct the mass loss history of NGC 6302 , including the geometry and composition of the AGB wind, infrared spectroscopy and imaging are needed. Unfortunately, the ISO data lack spatial information, limiting the analysis to the bulk dust composition. Mid-infrared imaging can reveal the present-day geometry of the dust envelope, which puts limits on the mass loss history.

This paper is organized as follows: In Sect. 2 the groundbased mid-infrared imaging and spectroscopy is presented. The ISO spectroscopy is discussed in Sect. 3, which describes our laboratory data of carbonates (Sect. 3.1) and a model fit to the observed spectrum (Sect. 3.2). In Sect. 4 we propose a possible geometry of NGC 6302 and discuss the astronomical relevance of carbonates. Our results are summarized in Sect. 5.

\section{The TIMMI2 observations}

\section{1. $N$ - and $Q$-band imaging}

We have observed NGC 6302 using the TIMMI2 mid-infrared imaging spectrograph attached to the $3.6 \mathrm{~m}$ telescope at the European Southern Observatory, La Silla, Chile. For a description of the instrument see Reimann et al. (2000). The observations were carried out on the night of June 17/18, 2001. Observing conditions were not optimal, with occasional clouds and variable seeing (between about $0.6^{\prime \prime}$ and $1.4^{\prime \prime}$ ). Imaging was done in four photometric bands, centred at 9.8 $(N 2), 10.4,11.9$, and $20(Q) \mu \mathrm{m}$. In addition, we obtained two 8.35-11.55 $\mu \mathrm{m}$ long-slit spectra. We observed HD 81797 and HD 169916 as point sources in order to determine the shape of the point spread function, as well as for flux calibration purposes, which is only performed in case of spectroscopy.

To correct for background, chopping and nodding has been performed. Apart from the $Q$-band image, the chopping and nodding images fell all inside the frame. For the various $N$-band images we used a lens scale corresponding to a pixel size of $0.3^{\prime \prime} /$ pixel, whereas the $Q$-band image was obtained with a pixel size of $0.2^{\prime \prime} /$ pixel. The dimensions of the detector are $320 \times 240$ pixels of which the central $300 \times 220$ pixels were used for further analysis. The chop throw used for the images in the $N$-band is $30^{\prime \prime}$, with a nodding offset of $30^{\prime \prime}$ perpendicular on the chop direction. For the $Q$-band image, we applied a chop throw of $40^{\prime \prime}$, and a nodding offset of $40^{\prime \prime}$ in the same direction as the chop.

Both the positive and negative images were used for the resulting image. The final image has been sharpened by means of a deconvolution using the point spread function (PSF) derived from HD 81797 for the $Q$-band. Unfortunately, for the $N 2,10.4$ and 11.9 filters no standard star was measured in the same night with the same settings, and we used observations of standards of another night. For the N2 and 10.4 filters no standards were available at all. Therefore, we used HD 169916 measured with the $11.9 \mu \mathrm{m}$ filter for all three filters for the PSF, assuming that the PSF would be similar in all three $N$-bands. A comparison with other N2-band images (with different settings, but the positive and negative images did overlap each other) showed that our assumption is not unreasonable.

Figure 1 shows the final TIMMI2 images at $N 2, N 10.4$, $N 11.9$, and $Q$ band, as well as the "raw" (i.e. before deconvolution) N2 image, and an optical VLT image of the nebula for comparison (ESO 1998). The "raw" N2 image shows a bright elongated region of approximately $10^{\prime \prime}$ by 4 ", and a much fainter region offset to the south and west. There is also evidence for faint arcs of emission that are reminiscent of the $\mathrm{X}$-shaped nebula seen in the optical image. In fact, the midIR emission has a morphology which is strikingly similar to that seen in the optical (see Fig. 2). This is surprising since the mid-IR emission originates from (warm) dust, while the optical emission is dominated by ionised gas. The equatorial torus, seen as a waist in the optical images, must contain very cold dust which does not yet contribute to the flux at $10 \mu \mathrm{m}$. We will return to this point when we discuss the ISO spectrum.

The deconvolved $N 2, N 10.4$ and $11.9 \mu \mathrm{m}$ images in Fig. 1 are very similar and show a rather "blobby" appearance. Note that the faint structure in the background is a common artifact of image deconvolution. However, the structure in the central area is real, since we see similar substructure in different observations deconvolved with different point spread functions. Also the $6 \mathrm{~cm}$ radio map of NGC 6302 shows substructure in this region (Gómez et al. 1989). Our images are very similar to the $3.3 \mu \mathrm{m}$ image taken by Casassus et al. (2000), taking into account that the orientation of their image is accidentally rotated by $180^{\circ}$ (Casassus, priv. comm.).

The deconvolved $Q$ band image (Fig. 1) tends to show more spherical emission, although the eastern part of the nebula is still dominating the emission. Due to the reduced spatial resolution at $20 \mu \mathrm{m}$ it is not possible to say if the emission is equally blobby as seen at $10 \mu \mathrm{m}$. 

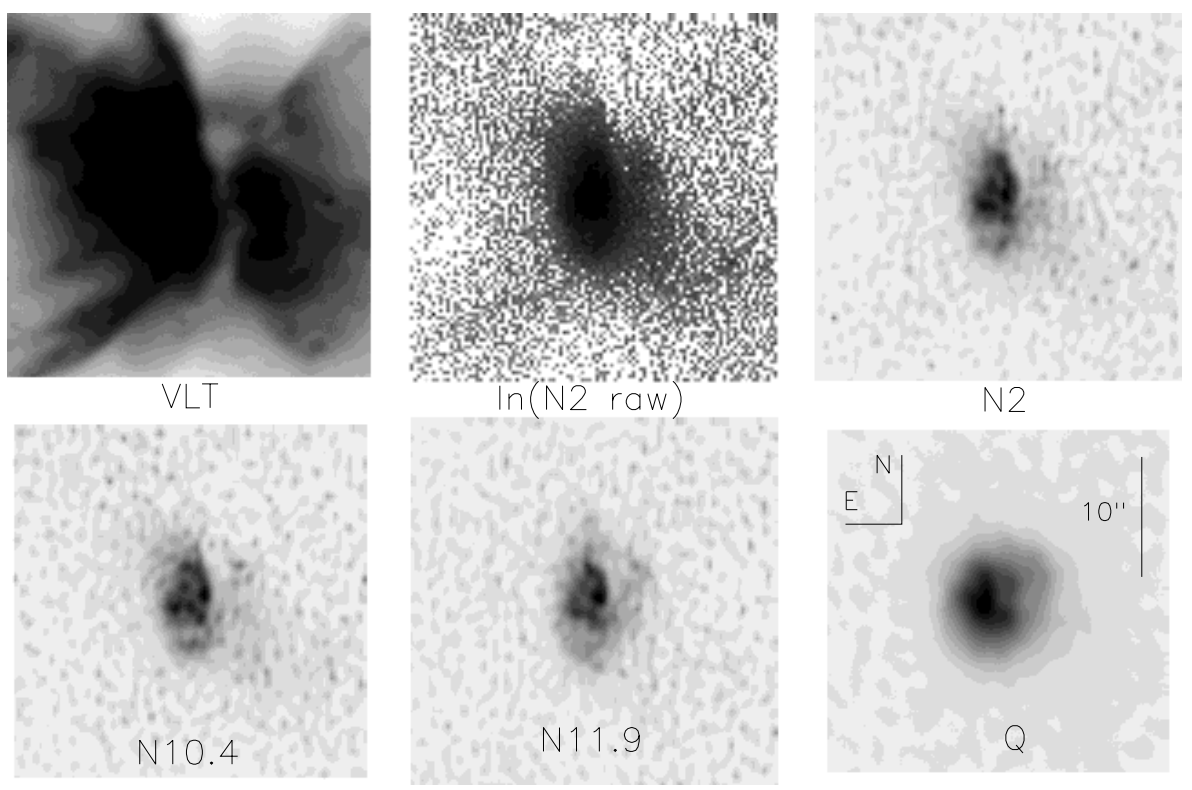

Fig. 1. TIMMI2 images of NGC 6302. In the upper left corner the VLT-first light image of NGC 6302 is shown (ESO 1998). The upper middle image is the logarithmic nondeconvolved $N 2$ band image, which shows the low brightness structure somewhat better than the deconvolved images. In the upper right corner the deconvolved $N 2$ band image is shown, in which detailed structure in the centre appears. The bottom row shows the deconvolved 10.4, 11.9 and $Q$-band images. All images have the same orientation and scale.

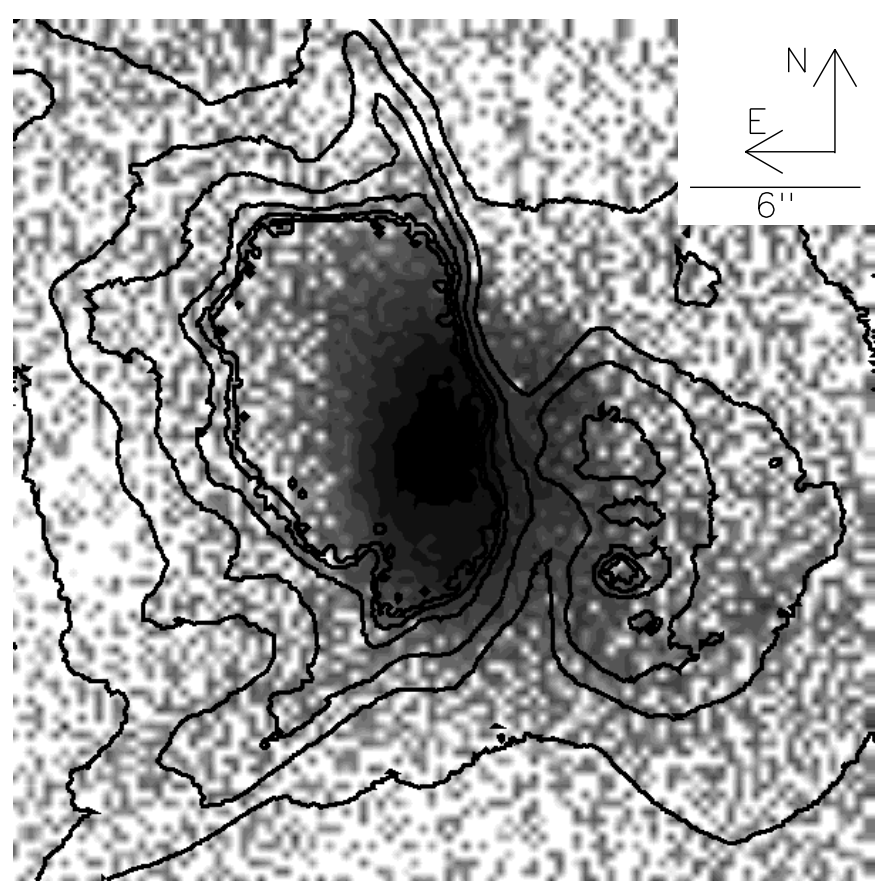

Fig. 2. TIMMI2 N2-band image of NGC 6302, overlaid with the contours of the optical VLT image (Fig. 1).

\subsection{N-band spectroscopy}

In Fig. 3 we show the spectra taken at the slit positions indicated in Fig. 4. We used HD 169916 as a reference spectrum, assuming that this star has a $F(v) \sim v^{2}$ flux distribution. The comparison with other calibration observations showed that the absolute flux calibration is only accurate up to about $40 \%$. The relative flux calibration is much more accurate. The wavelength calibration provided by ESO on the TIMMI2 home page appeared to be inaccurate, and we determined a new wavelength calibration, based on the [ArIII] and [SIV] line, assuming a linear pixel to wavelength dependence. The fact that the [Navi] line and the PAH features are now at the right

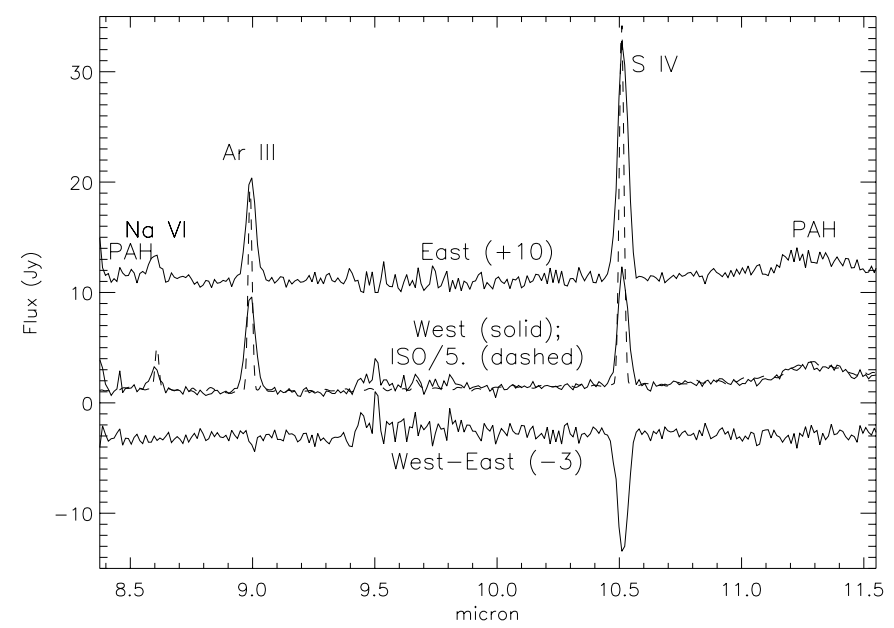

Fig. 3. TIMMI $N$-band spectra NGC 6302. The spectra were taken $1.2^{\prime \prime}$ east and 1.2" west of the brightest (east of the torus) lobe in the image. For comparison the ISO spectrum (divided by 5) is given as a dashed line. The relative flux calibration is quite accurate, but the absolute flux calibration is only certain up to $40 \%$. The difference spectrum of the west and east side of the lobe is also given. The lines around $9.5 \mu \mathrm{m}$ are due to telluric lines.

wavelengths shows that our assumption is valid (using the calibration files provided by ESO the $11.3 \mu \mathrm{m}$ PAH feature was found at $11.7 \mu \mathrm{m})$. The comparison with the ISO spectrum (dashed line in Fig. 3), gives confidence in the new wavelength calibration.

The spectrum is dominated by the forbidden emission lines of [SIv] and [ArIII. However the PAH features at 11.3 and also $8.6 \mu \mathrm{m}$ are also found. The noise around $9.5 \mu \mathrm{m}$ is due to telluric lines.

The east and west slit are both probing the eastern lobe of the nebula (Fig. 4). The difference spectrum is very flat, apart from the [SIv] line. The fact that even around 8.6 and $11.3 \mu \mathrm{m}$ it is flat, indicates that the distribution of the PAHs is quite 


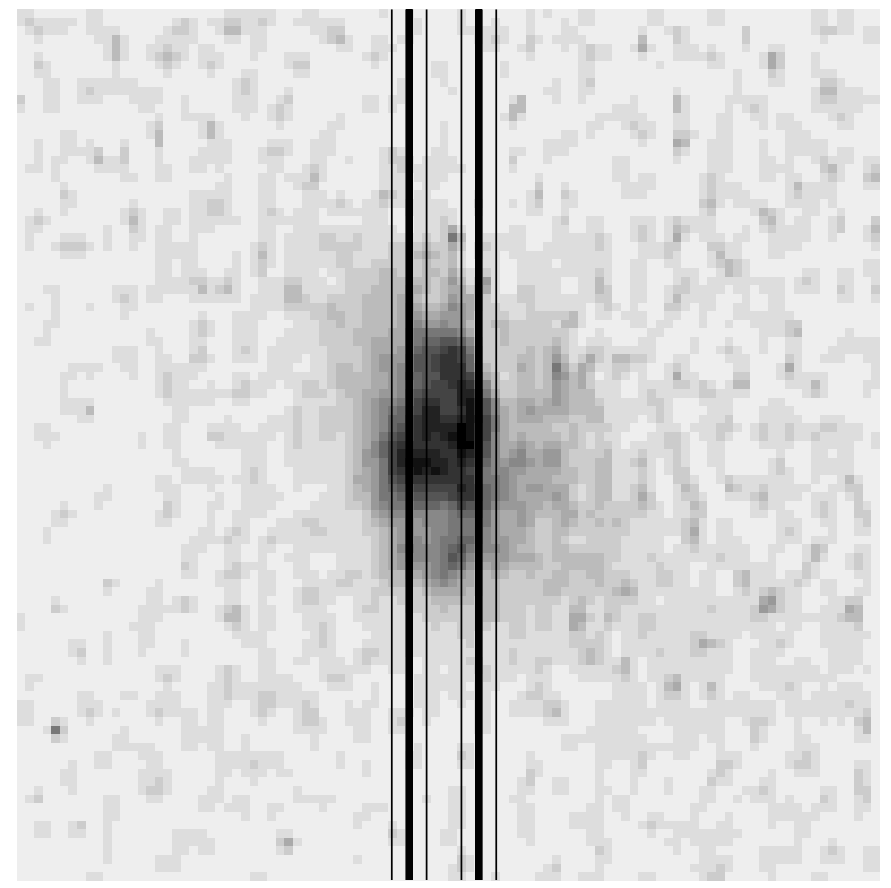

Fig. 4. The position of the $N$-band slit showed on the deconvolved $N 2$ image. North is up and east is to the left. Both slits were $1.2^{\prime \prime} \times 70^{\prime \prime}$ and placed 1.2" east and west from the central peak. It is clear that both slit positions cover the eastern lobe of the nebula.

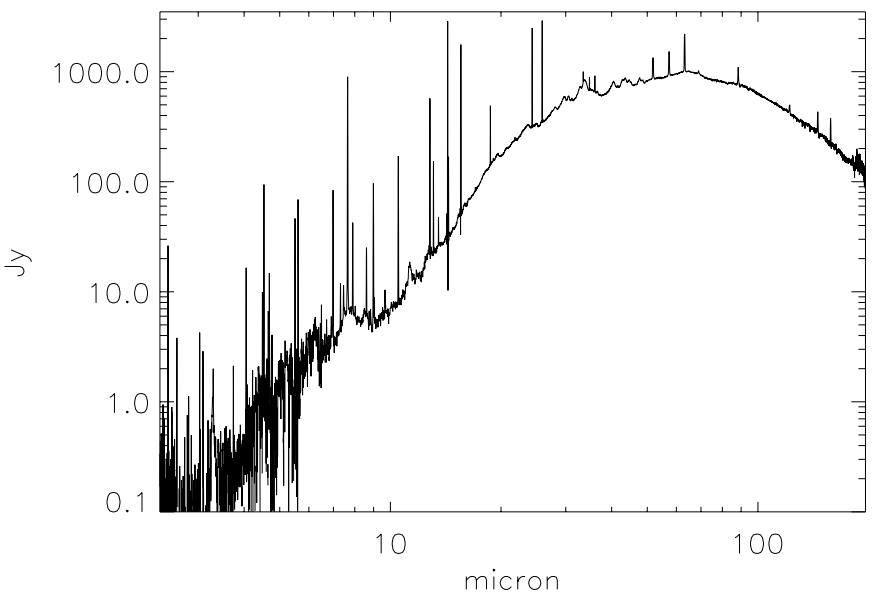

Fig. 5. The ISO 2.4-200 $\mu \mathrm{m}$ spectrum of NGC 6302. This figure is based on data presented in Molster et al. (2001a). Clearly visible are the numerous emission lines due to fine-structure transitions in the ionized gas.

constant in the bright eastern lobe. This is in agreement with the $3.3 \mu \mathrm{m}$ image obtained by Casassus et al. (2000).

\section{Analysis of the ISO spectrum}

The ISO SWS and LWS spectra have been presented by Barlow (1998) and Molster et al. (2001a, 2002b) and in Paper I. We briefly repeat the overall characteristics of the spectrum. The SWS and LWS apertures, which have sizes in the range $14 \times 20^{\prime \prime}$ to $80^{\prime \prime}$, cover a large fraction of the nebula and therefore provide no detailed spatial information. The spectrum, shown in Fig. 5, is dominated by a strongly rising spectrum
Table 1. Chemical formula, crystal system and content of impurities of the carbonate minerals determined by SEM and EDX measurements. The impurities in dolomite, magnesite, siderite and ankerite are the carbonates corresponding to the metals mentioned in the last column. The used abbreviations in Col. 3 describe the crystal system of the mineral (trigonal and rhombic).

\begin{tabular}{lccl}
\hline \hline Mineral & Formula & $\begin{array}{c}\text { Crystal } \\
\text { system }\end{array}$ & $\begin{array}{l}\text { Impurities } \\
\text { (wt\%) }\end{array}$ \\
\hline dolomite & $\mathrm{CaMg}\left(\mathrm{CO}_{3}\right)_{2}$ & tri. & $\sim 0.5 \mathrm{Fe}$, very pure \\
calcite & $\mathrm{CaCO}_{3}$ & tri. & $<0.1 \mathrm{Si}$, very pure \\
aragonite & $\mathrm{CaCO}_{3}$ & rhom. & $<0.2 \mathrm{Si}$, very pure \\
magnesite & $\mathrm{MgCO}_{3}$ & tri. & $\sim 1.0 \mathrm{Fe} ; 0.2-0.9 \mathrm{Ca}$ \\
siderite & $\mathrm{FeCO}_{3}$ & tri. & $\sim 2.0 \mathrm{Mg} ; \sim 5.0 \mathrm{Mn}$ \\
ankerite & $\mathrm{CaFe}\left(\mathrm{CO}_{3}\right)_{3}$ & tri. & $2-3 \mathrm{Mn} ; 3.5-4.2 \mathrm{Mg}$ \\
\hline
\end{tabular}

which peaks near $55 \mu \mathrm{m}$, and numerous, sometimes rather narrow emission bands. Both the continuum and these emission bands are caused by the circumstellar dust. In addition, strong, spectrally unresolved emission from fine-structure lines are evident, which are caused by the ionized nebular gas. These lines can be used to determine the chemical abundance of the gas and properties of the central star (e.g. Pottasch et al. 1996; Pottasch $\&$ Beintema 1999). In this work we focus on the analysis of the dust component.

\subsection{Laboratory spectroscopy of carbonates}

Molster et al. (2001a) provide a useful inventory of the solid state emission bands in the ISO spectrum of NGC 6302, and we will not repeat their analysis here. While many emission bands were successfully allocated to (mostly oxygen-rich) dust components, several prominent emission bands, notably near 29, 48, 60 and $90 \mu \mathrm{m}$ remained partially unidentified. We have investigated possible carriers for the $\sim 92 \mu \mathrm{m}$ band, reported to be present in NGC 6302 and NGC 6537 (Molster et al. 2001a, $2002 b)$. During the course of our laboratory studies of cosmic dust analogues, we noted that the carbonate calcite $\left(\mathrm{CaCO}_{3}\right)$ has a strong resonance at $\sim 92 \mu \mathrm{m}$. This prompted us to carry out a systematic laboratory study of transmission spectra of several carbonate species. A series of carbonates of possible cosmic relevance has been chosen for laboratory investigations, including the natural minerals ankerite (origin unknown), aragonite (Bohemia), dolomite (Eugui, Navarra, Spain), calcite (origin unknown), magnesite (Steiermark, Austria) and siderite (Schönbrunn, Vogtland, Germany). The purity and homogeneity of the natural samples are investigated by scanning electron microscopy (SEM) and energy dispersive X-ray analysis $(\mathrm{EDX})$. The macroscopic phase homogeneity of the carbonate samples was measured by X-ray diffraction. The chemical composition and the content of impurities are presented in Table 1.

The minerals dolomite, calcite, aragonite and magnesite turned out to be very pure and homogeneous. The impurities in siderite and ankerite are the corresponding carbonates mentioned in Table 1. These are homogeneously distributed within the main mineral component. 


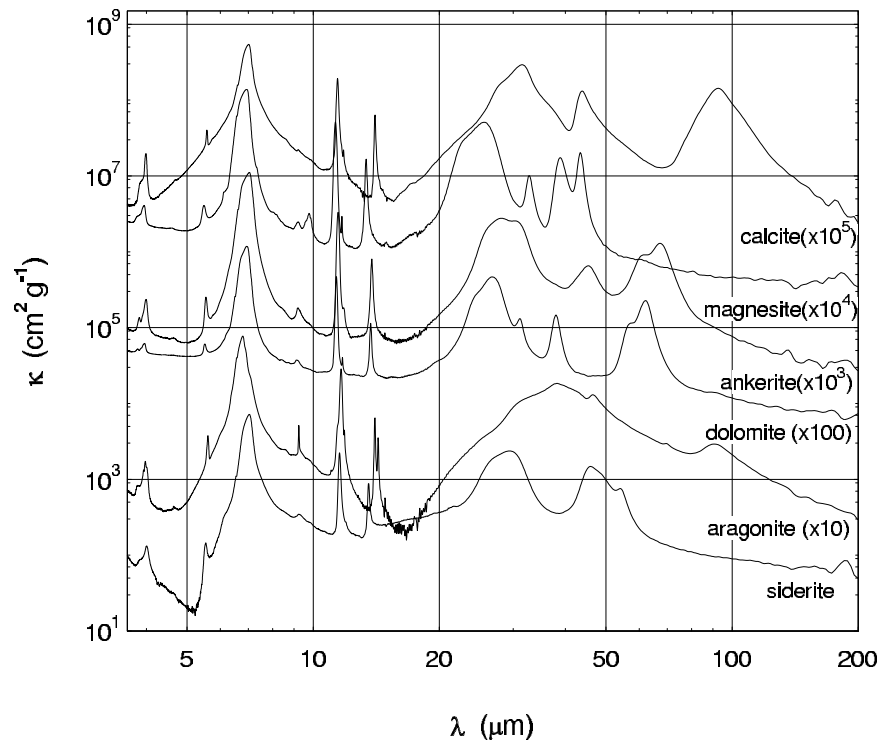

Fig. 6. Mass absorption coefficients of natural carbonates derived from transmission measurements of the powders embedded in $\mathrm{KBr}$ and $\mathrm{PE}$. For clarity, the spectra have been multiplied by the factor indicated in the plot.

Small carbonate grains with an average size smaller than $2 \mu \mathrm{m}$ have been produced by grinding and sedimentation in water-free acetone. In order to measure the infrared spectrum over a wide spectral range the small grains were embedded in transparent material like $\mathrm{KBr}$ and Polyethylene (PE). Infrared spectroscopy was performed using a Bruker $113 \mathrm{v}$ Fourier transform spectrometer in the wavelength range between 2-200 $\mu \mathrm{m}$. The infrared spectral behaviour of the carbonate minerals is demonstrated in Fig. 6, and can be found in digitized form in the Jena-St. Petersburg Database of Optical Constants (Henning et al. 1999).

Magnesite, siderite, ankerite, dolomite, and calcite belong to the carbonates of the calcite group and crystallize in the trigonal crystal system. Carbonates containing cations with larger radii than $\mathrm{Ca}^{2+}$ crystallize in the rhombic crystal system. $\mathrm{CaCO}_{3}$ is dimorph and can exist in both structures as calcite and aragonite. The typical crystalline structure of the carbonates in the calcite group is characterized by an alternating sequence of cation and anion layers. In both magnesite and calcite, the plane carbonate group consists of three oxygen located at the corners of an equilateral triangle and a carbon in the centre. The bonding between $\mathrm{C}$ and $\mathrm{O}$ is covalent whereas the bonding between the cations and the carbonate anion $\left(\mathrm{CO}_{3}{ }^{2-}\right)$ is mainly ionic. Each cation is octahedrally coordinated by six $\mathrm{O}$ atoms of the adjacent $\mathrm{CO}_{3}{ }^{2-}$ ion. The crystals of the calcite group with mixed metal composition - like dolomite and ankerite - show an alternating arrangement of the calcium and magnesium or calcium and iron layers. In contrast to the calcite structure the $\mathrm{Ca}^{2+}$ ion in aragonite is coordinated by $9 \mathrm{O}$-atoms.

The infrared optical properties are determined by the crystalline structure of the minerals. The spectral behaviour of carbonates has been measured several times in previous studies (e.g. Hellwege et al. 1970; Penman 1976; Orofino et al. 1997; Yamagishi et al. 2001). The measured mid-infrared bands arise mainly from the carbonate anion. The planar structure of this anion and its $\mathrm{C}_{3}$ symmetry gives rise to three infrared active modes at about 7,11 and $14 \mu \mathrm{m}$. The $7 \mu \mathrm{m}$ band can be attributed to the $\mathrm{C}=\mathrm{O}$ stretching mode whereas the 11 and $14 \mu \mathrm{m}$ bands are caused by the out-of-plane and in-plane bending modes. The low frequency bands beyond $25 \mu \mathrm{m}$ are due to translation of the metal cations and represent motions perpendicular and parallel to the plane of the carbonate anions (Hellwege et al. 1970).

\subsection{Dust model fit}

\subsubsection{Description of the model}

We use the identified solid state components to quantitatively fit the entire ISO spectrum. Inspection of the ISO data shows that all dust bands are in emission, suggesting that the dust shell is optically thin at infrared wavelengths. This substantially simplifies the analysis. Given the complex geometry of the nebula, the multiple dust components present in the spectrum, and the still incomplete knowledge of the dust species present, we decided to focus our analysis on determining the relative mass contributions of the known dust species, rather than attempting a full 2-dimensional radiative transfer calculation. We use the optically thin nature of the dust at mid- and far-infrared wavelengths to constrain the dust mass of the components using mass absorption coefficients. The thus derived dust masses will not depend on the assumed geometry as long as the emission is optically thin. The problem then reduces to deriving the temperature over mass distribution of the various dust components (e.g. Bouwman et al. 2000). The advantage of using mass absorption coefficients in an optically thin dust shell, is that it does not require knowledge of the location of the dust.

We derive the dust mass and temperature distribution under the following assumptions: i) the dust shell is optically thin at mid- and far-infrared wavelengths; ii) all grains have the same size, i.e. $0.1 \mu \mathrm{m}$; iii) all grains are spherical; iv) all grains are of homogeneous composition; v) the number density of dust grains as a function of distance is a simple power law (see Eq. (4), where $\rho_{0}=\frac{4}{3} \pi a^{2} \rho_{\mathrm{d}} n_{0}$ ); vi) the dust species all have the same temperature ranges, limited by the temperatures at the inner radius $T_{0}$ and at the outer radius $T_{\max }$, which are free parameters. The result of our analysis is a mass over temperature distribution $M_{\mathrm{d}}(T)$ of all identified dust species. In Sect. 4.1.1 we will discuss the validity and the impact of these assumptions.

In the general case of an optically thin dust cloud, which specifically applies to an optically thin dust shell, the specific intensity emitted by the dust in the cloud can be written as

$I_{v}(T)=\int_{V} \alpha_{v} B_{v}(T) \mathrm{d} V \quad$ for $\quad \tau \ll 1$

where $\alpha_{v}$ is the specific absorptivity. By integrating over volume $V$ this can be written as

$I_{v}(T)=N \sigma_{v} B_{v}(T) \quad$ or $\quad I_{v}(T)=M_{\mathrm{d}}(T) \kappa_{v} B_{v}(T)$

with the absorption cross-section can be described in terms of the geometric cross-section and absorption efficiency, according to $\sigma_{v}=\sigma_{\text {geom }} Q_{v}$. Following for instance 
Bouwman et al. (2000), $M_{\mathrm{d}}(T)$ is the dust mass at temperature $T$. The mass absorption coefficient is given by $\kappa_{v}$ and the number of grains in the volume is represented by $N$.

When we realize that we can write for the specific flux $F_{v}=I_{v} / D^{2}$, and under the assumption that the dust grains are identical spherical grains with a radius $a$, we find

$F_{v}(T)=\frac{1}{D^{2}} \frac{1}{\rho_{\mathrm{d}}} \frac{3}{4} \frac{1}{a} M_{\mathrm{d}}(T) Q_{v} B_{v}(T)$

with $D$ the distance to the dust cloud and $\rho_{\mathrm{d}}$ the density of the material in the dust grain.

In case of a circumstellar dust shell, we may assume that the temperature and density distribution follow a power law:

$T(r)=T_{0}\left(\frac{r}{r_{0}}\right)^{-q} \quad \rho(r)=\rho_{0}\left(\frac{r}{r_{0}}\right)^{-p}$

where $r$ represents the distance to the central star, and $r_{0}$ the inner radius of the dust shell. Using $\mathrm{d} M_{\mathrm{d}}=4 \pi r^{2} \rho(r) \mathrm{d} r$, we can express $M_{\mathrm{d}}$ in terms of the temperature distribution in the dust shell, according to

$M_{\mathrm{d}}(T(r))=\frac{4 \pi \rho_{0} r_{0}^{3}}{3-p}\left(\frac{T(r)}{T_{0}}\right)^{-\frac{3-p}{q}}$.

For spherical dust grains the density at the inner radius is $\rho_{0}=$ $\frac{4}{3} \pi a^{3} \rho_{\mathrm{d}} n_{0}$, with $n_{0}$ the dust grain number density at the inner radius, which is different for each identified dust component. The density $\rho_{\mathrm{d}}$ corresponds to the density of the grain material itself. This can be combined with Eqs. (5) and (3) to obtain an expression of the specific flux:

$F_{v}(T)=\frac{1}{D^{2}} \frac{4 \pi a^{2} r_{0}{ }^{3} n_{0}}{3-p}\left(\frac{T(r)}{T_{0}}\right)^{-\frac{3-p}{q}} Q_{v} B_{v}(T)$

where the observable total specific flux $\mathcal{F}_{v}$ is given by

$\mathcal{F}_{v}=\int_{T_{0}}^{T_{\max }} F_{v}(T) \mathrm{d} T$

which can be compared directly to the ISO spectroscopy.

\subsubsection{Fit parameters and dust composition}

Following the analysis of Molster et al. (2001a), we assumed that enstatite $\left(\mathrm{MgSiO}_{3}\right)$, forsterite $\left(\mathrm{Mg}_{2} \mathrm{SiO}_{4}\right)$ and crystalline water ice are present, in addition to amorphous silicates. Diopside $\left((\mathrm{Ca}, \mathrm{Mg}) \mathrm{SiO}_{3}\right)$ is believed to contribute to the $60 \mu \mathrm{m}$ complex (Koike et al. 2000). Diopside is in the form of a solid solution between wollastonite $\left(\mathrm{CaSiO}_{3}\right)$ and enstatite $\left(\mathrm{MgSiO}_{3}\right)$ in the number ratio 46:54, i.e. Wo46En54. In addition, we considered metallic iron as a dust component, which is found to be present in the dust shells of evolved stars (Kemper et al. 2002a), and the carbonate species presented in this work. Under the assumption that all dust grains are spherical and have a radius $a$ of $0.1 \mu \mathrm{m}$, we have derived $Q_{v}$ values from laboratory measurements of amorphous olivine (Dorschner et al. 1995); metallic iron (Henning \& Stognienko 1996); forsterite and clino-enstatite (Koike et al. 1999); diopside (Koike et al. 2000); water ice (Bertie et al. 1969; Warren 1984) and carbonates (this work).

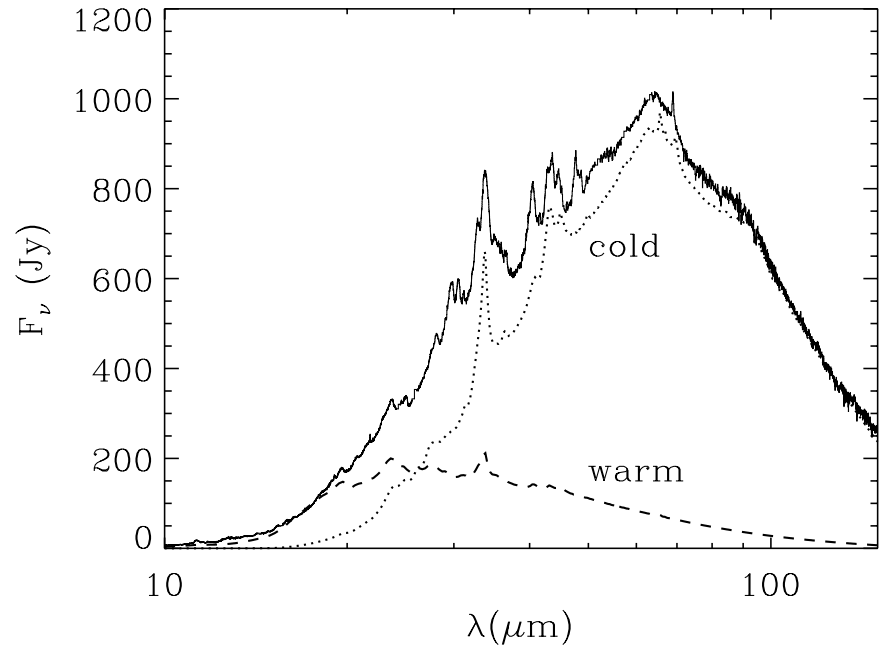

Fig. 7. The warm and cool dust components required to fit the ISO spectrum of NGC 6302. The solid line represents the ISO spectrum. The warm (100-118 K) and cool (30-60 K) dust components are represented by the labelled dotted and dashed lines.

Using Eqs. (6) and (7) the far-infrared spectrum has been fitted, under the assumption that all dust species are found in the same temperature regimes. Thus, the free parameters to be fitted are the number density at the inner radius $n_{0}$, which differs for each dust component and scales with the mass contained in that component; the temperature range defined by $T_{0}$ and $T_{\max }$; and the slopes $p$ and $q$ of the density and temperature power laws. For the distance $D$ we have used the measured value of $0.91 \mathrm{kpc}$ (Shaw \& Kaler 1989) and the grain size $a$ is assumed to be $0.1 \mu \mathrm{m}$. The inner radius $r_{0}$ follows from the values of $T_{0}$ and $q$, and is therefore not a fit parameter. If $p$ and $q$ are both chosen to be $-1 / 2$ the best results are obtained. Inspection of the spectrum shows that the temperature distribution of the dust must be very wide, which is incompatible with a simple $r^{-2}$ density distribution of the dust. We attempted to fit the spectrum with a single range of temperatures but were not successful; apart from a cool dust component (30-60 K), peaking near $55 \mu \mathrm{m}$, we had to introduce a second dust component at 100-118 K dominating the spectrum between 10 and $30 \mu \mathrm{m}$ (see Fig. 7). The results of the fits are shown in Table 2, where the mass of each dust component for both temperature ranges is given. These masses are constrained by $n_{0}$ which is separately fitted for all identified dust species and both temperature ranges, and are obtained by integrating Eq. (5) over the considered temperature range.

The warm dust component could not consist of amorphous olivine, because the strong resonances due to the $\mathrm{Si}-\mathrm{O}$ stretching and $\mathrm{O}-\mathrm{Si}-\mathrm{O}$ bending modes at 9.7 an $18 \mu \mathrm{m}$ are not observed. Iron is used as the carrier of the warm continuum, but as PAHs have been detected in NGC 6302 (Molster et al. 2001a), amorphous carbon, instead of iron, could also explain the continuum emission. A mixed chemistry is not uncommon for evolved stars, it is for example seen in IRAS 09425-6040 (Molster et al. 2001b). We conclude that the composition of the warm dust is different from the composition of the cool dust. 
Table 2. Empirically derived dust masses by fitting the ISO spectrum of NGC 6302 for the temperature regimes 30-60 K and 100-118 K. The dust masses are obtained by integrating Eq. (5) over the considered temperature range. The absolute accuracy of the dust masses is a factor of three, this is due to the uncertainties in laboratory measurements and inaccuracies in the used assumptions. The relative dust masses, i.e. the mass ratios between the various identified species, are estimated to have an error of $\sim 50 \%$ (see Sect. 4.1.1). The values for the crystalline silicate components are lower limits, because we used laboratory measurements that were intrinsically much stronger than similar measurements obtained by other groups (Sect. 4.1.1). In cases where lower limits are given, we did not detect the species indicated. The value indicates the maximum amount that could be present without showing the spectral features at a discernible level.

\begin{tabular}{lccccc}
\hline \hline species & $T(\mathrm{~K})$ & $M_{\mathrm{d}} / M_{\odot}$ & mass fraction & $T(\mathrm{~K})$ & $M_{\mathrm{d}} / M_{\odot}$ \\
\hline $\begin{array}{l}\text { amorphous olivine } \\
\text { iron (or carbon) }\end{array}$ & $60-30$ & $4.7 \times 10^{-2}$ & $94 \%$ & $118-100$ & $6.1 \times 10^{-6}$ \\
forsterite & & & & $118-100$ & $1.2 \times 10^{-4}$ \\
clino-enstatite & $60-30$ & $>2.0 \times 10^{-3}$ & $>4.0 \%$ & $118-100$ & $3.7 \times 10^{-7}$ \\
water ice & $60-30$ & $>5.5 \times 10^{-4}$ & $>1.1 \%$ & $118-100$ & $3.1 \times 10^{-7}$ \\
diopside & $60-30$ & $3.6 \times 10^{-4}$ & $0.72 \%$ & $118-100$ & $<1.5 \times 10^{-8}$ \\
calcite & $60-30$ & $2.8 \times 10^{-4}$ & $0.56 \%$ & $118-100$ & $<1.2 \times 10^{-7}$ \\
dolomite & $60-30$ & $1.3 \times 10^{-4}$ & $0.26 \%$ & $118-100$ & $<1.0 \times 10^{-7}$ \\
\hline
\end{tabular}

The exact composition of the cold amorphous dust is unknown. Amorphous olivine is used to produce the required continuum emission, but other abundant species with a smooth absorption spectrum at far-infrared wavelengths, such as pyroxene and iron, could be contributing as well. The mass fractions of forsterite and enstatite are lower limits. We used the laboratory data leading to the best spectral match with the observations, i.e. those of Koike et al. (1999).

The previously unidentified $92 \mu \mathrm{m}$ band (Molster et al. 2001a) can be fitted very accurately with calcite $\left(\mathrm{CaCO}_{3}\right.$, trigonal), see Fig. 8. The strong resonances of calcite at shorter wavelengths (see Fig. 6) are not discernible in the ISO spectrum, indicating that it is not a significant component in the warm dust. Aragonite $\left(\mathrm{CaCO}_{3}\right.$, rhombic) also has a resonance at $\sim 92 \mu \mathrm{m}$, and may therefore contribute to the observed feature in NGC 6302. However it is not very likely to exist in a Planetary Nebula. Although it has the same chemical composition as calcite, its lattice structure is different, and it can only exist under relatively high pressure and temperature.

The $60 \mu \mathrm{m}$ feature can be accurately fitted using absorption efficiencies of dolomite, diopside and water ice (see Fig. 9). Ankerite $\left(\mathrm{FeCO}_{3}\right)$ has a resonance at approximately the same wavelength (see Fig. 6) and could in principle contribute to the $60 \mu \mathrm{m}$ complex. However, solar system carbonates are almost exclusively in the form of dolomite and calcite (Johnson \& Prinz 1993; Brearley \& Jones 1998) and therefore, we concentrated our analysis on dolomite.

The results of our model fit are shown in Fig. 8 and Table 2. Since the dust shell is optically thin, the relative mass fractions can be derived from the model fit. The absolute dust mass of each mineralogical component scales with the distance, and if $910 \mathrm{pc}$ is adopted for the distance (Shaw \& Kaler 1989), we arrive at the dust masses as indicated in Table 2. We derived that the depletion of calcium into the identified calcium bearing species - calcite, dolomite and diopside - is about $30 \%$ (Paper I), if we assume a dust/gas mass ratio of $1 / 100$ and adopt a calcium abundance of $[\mathrm{Ca} / \mathrm{H}]=2.2 \times 10^{-6}$ (Snow 1984). From the fit to the $60 \mu \mathrm{m}$ complex the fraction of water contained in the solid phase can be calculated. Adopting a mass ratio of $1 \times 10^{-3}$ between $\mathrm{H}_{2} \mathrm{O}$ and $\mathrm{H}_{2}$
(González-Alfonso \& Cernicharo 1999) we find that $10 \%$ of the water is contained in water ice. This is consistent with the results of Hoogzaad et al. (2002). The presence of $\mathrm{OH}$ molecules (Payne et al. 1988) indicates that water should also be present in the vapour phase.

\section{Discussion}

\subsection{Geometry and composition of the circumstellar dust shell}

In Sect. 3.2.2 we derived that the dust in the circumstellar environment of NGC 6302 consists of two temperature components. The cold component (30-60 K) has a mass of $0.050 M_{\odot}$ and contains silicates, water ice and carbonates, indicative of a oxygen-rich chemistry. The warm component (100-118 K) contains only $1.3 \times 10^{-4} M_{\odot}$, which is mainly caused by a featureless species like carbon or iron. About 5\% of the warm component is in the form of silicates. The large difference in chemical composition between the warm and the cold component and the lack of continuity in the temperature distribution suggest that the two components have a different spatial distribution. This then allows a mixed chemistry; regions of C-rich dust could exist in a PN with a strong silicate spectroscopic signature. The presence of PAHs in NGC 6302 (first reported as UIR bands by Roche \& Aitken 1986) supports the idea of a mixed chemistry, both the warm, diffuse component and the PAHs could reside in the same regions of the nebula.

The large mass of the cold component suggests that it is present in a circumstellar torus, which leaves the polar regions for the warm carbon-rich component. Optical images of NGC 6302 show that the eastern lobe is brighter than the western lobe (see Fig. 1 and also Schwarz et al. 1992), suggesting that the western lobe is partly obscured by the circumstellar torus. This leads to a geometry as indicated in Fig. 10. The dusty torus around the central star is seen under an inclination angle $\sim 45^{\circ}$, and partially obscures the western lobe. In return, the eastern part of the torus is located behind the eastern outflow. The dusty torus contains a high column density of dust towards the central star, and therefore, most of the dust is effectively shielded from heating by the central star and remains 


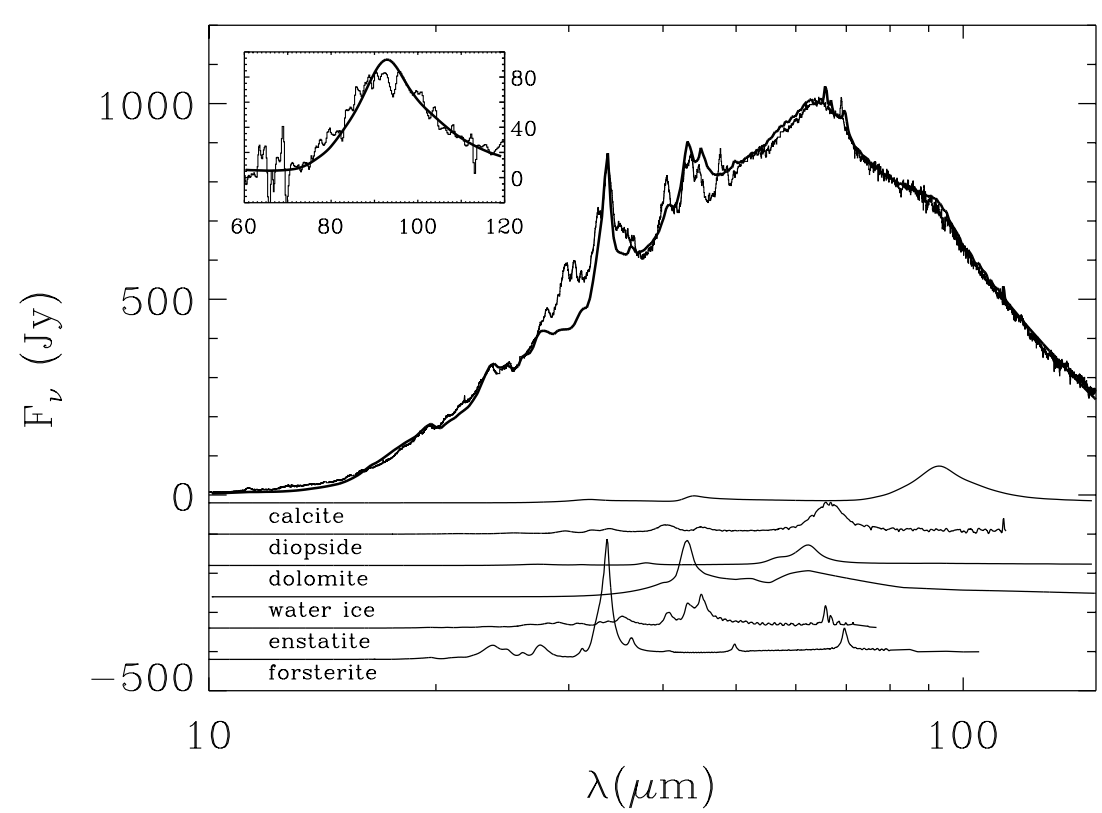

Fig. 8. Model fit to the ISO spectrum of NGC 6302. The thin line represents the observed spectrum, the fit, consisting of the components given in Table 2, is indicated with the thick line. In the lower part of the plot, the contributions of the cold dust components (30-60 K) are indicated, except the contribution of amorphous olivine, which leads to a smooth overall contribution. The inset shows the observed spectrum (thin line) and the model fit (thick line) from $60-120 \mu \mathrm{m}$, where the contributions of all species, except calcite, are subtracted from both the observations and the model. Thus, it only shows the observed and modelled calcite $92 \mu \mathrm{m}$ feature. Figure adopted from Paper I.

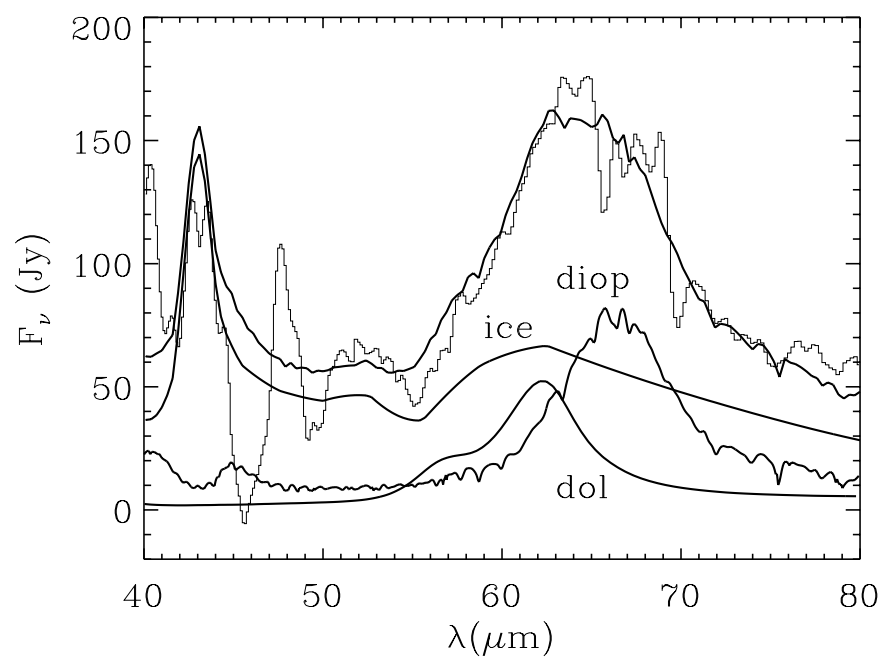

Fig. 9. Contributions to the $60 \mu \mathrm{m}$ complex in NGC 6302. The thin line represents the ISO spectrum of NGC 6302 from $40-80 \mu \mathrm{m}$ with all contributions subtracted, except the contributions of diopside, dolomite and water ice. The thick lines represent the contributions of diopside, water ice and dolomite to $F_{v}$, indicated with abbreviations of their mineral names. The uppermost thick line is the sum of the contributions of diopside, dolomite and water ice.

relatively cool. The dust in the polar regions however, is directly radiated by the central star and thus has a higher temperature.

Our observations, as well as previously published work, is in agreement with the proposed geometry. The torus is optically thick in the UV, optical and near-infrared. Therefore, the optical images only show the scattered light from the central star in the polar regions. We have convolved the warm and cool dust spectra modelled in Sect. 3.2.2 with the TIMMI2 N11.9and $Q$-band transmission profile and corrected for the atmospheric transmission in order to determine the contribution of the dust components in both bands. We find that in the N11.9band, only $0.2 \%$ of the flux comes from the cold component,

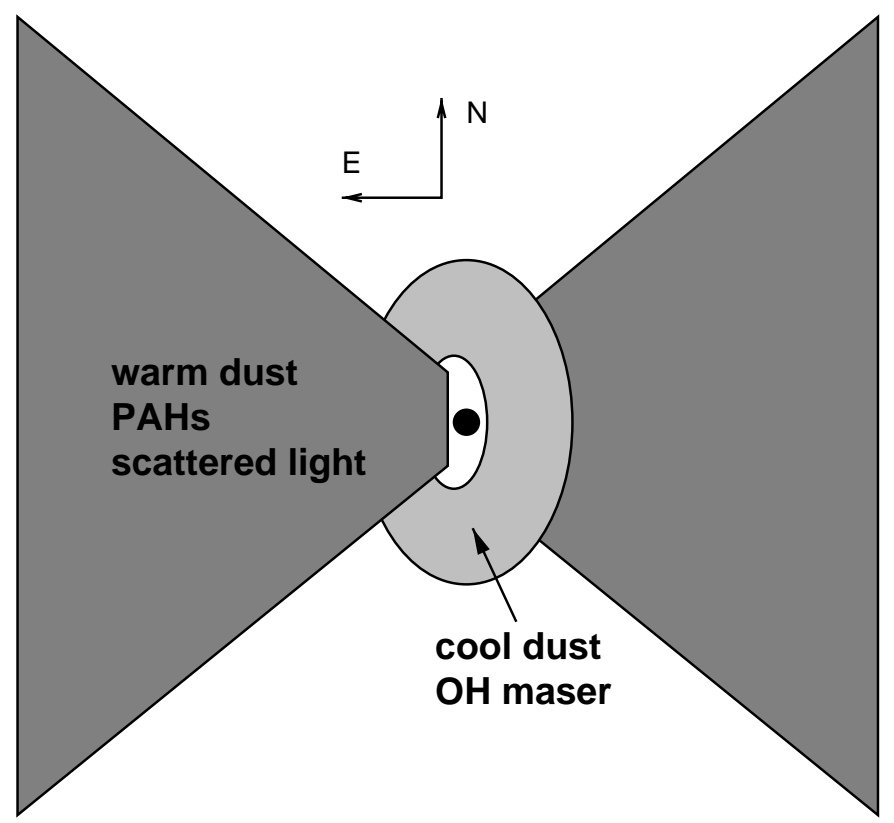

Fig. 10. Proposed geometry of NGC 6302. A dense torus of cool dust is present around the central star (light grey) under an inclination of $45^{\circ}$ with respect to the line-of-sight. More diffuse material in the polar regions, is indicated in dark grey. The torus obscures the polar region on the western side. A part of the torus is located behind the eastern lobe. The emission from the warm dust and PAHs originates from the lobes, as well as scattered light from the central star. The torus contains the cool dust and the $\mathrm{OH}$ maser. For clarity the approximate position of the central star is indicated, however in reality it is obscured by the dusty torus and can only be studied in scattered light from the polar regions.

and consequently $99.8 \%$ originates from the warm dust component. For the $Q$-band these numbers are $29.0 \%$ and $71.0 \%$ respectively. Since the peak in intensity in the $N$-band images and the optical image coincide (see Fig. 2) we conclude that indeed the warm dust is located in the polar regions. The $Q$ band imaging reveals a different picture; one third of the flux 
originates from the cool dust component, and two third from the warm dust. Both the warm dust in the eastern lobe, and the cool dust in the western part of the torus contribute to the observed image, leading to a more or less spherical structure in this wavelength band (Fig. 1).

$N$-band spectroscopy of the eastern lobe shows PAH emission. From Fig. 6 of (Casassus et al. 2000, figure is rotated by $180^{\circ}$ ) it is clear that the PAH emission intensity peak coincides with the intensity peak in the optical and $N$-band, indicating that the PAH emission also arises from the lobes, where UV radiation from the central star can penetrate. The UV radiation field also explains the ionization observed in the lobes (Fig. 3). Persi et al. (1999) also notice that the emission of warm dust grains and forbidden line transitions emerges from the same region. The $6 \mathrm{~cm}$ data presented by Gómez et al. (1989) traces the location HII region. In their Fig. 3 the region confined by the torus is clearly visible.

On the other hand, the optically thick torus around the central star effectively shields the molecular material in the torus from the high energy radiation. The infrared radiation field in the torus will pump $\mathrm{OH}$ molecules present and cause the formation of $\mathrm{OH}$ masers. Indeed, $\mathrm{OH}$ maser emission is observed in NGC 6302, on the west side of the object (Payne et al. 1988), indicating that it arises from part of the torus located at the front side of the object. This is consistent with the fact that predominantly the blue-shifted part of the line profile is seen (Payne et al. 1988).

\subsubsection{Validity of model assumptions}

The geometry described here is consistent with the assumptions we have used to fit the ISO spectrum, and with the TIMMI2 images. Here we will discuss the validity of the assumptions used in this model.

i) We assumed that the dust is optically thin at large wavelengths. The TIMMI2 images indeed suggest that this is true: the torus is still visible as a dark lane in the $N$-band images but starts to disappear when NGC 6302 is observed in the $Q$-band. At longer wavelengths, where the spectral energy distribution (SED) peaks and the different solid state species are identified, the dust torus will be optically thin.

A simplified calculation verifies this: The dust is thought to be present in a torus, with a scale height $h$ from the equatorial plane and an outer radius $R_{\text {out }}$. The inner radius is small compared to the outer radius and can be ignored. The torus is seen under an inclination angle of $45^{\circ}$ (Bohigas 1994). The length of the line-of-sight through the disk is thus $l=2 h \sqrt{2}$. Assuming that the density is constant throughout the disk, we find for the infrared optical depth along this line-of-sight:

$\tau_{\text {IR }}=Q n \sigma_{\text {geom }} 2 h \sqrt{2}$

where

$n=\frac{M_{\mathrm{d}}}{M_{\text {grain }}} \frac{1}{2 h \pi R_{\text {out }}^{2}}$.

By adding all cold dust components listed in Table 2, the dust contained in the torus is determined to be $M_{\mathrm{d}}=0.05 M_{\odot}$.
The mass of a grain follows from the used grain size of $0.1 \mu \mathrm{m}$, and the average density of silicate grains. Using cgs units, we find that the torus is optically thin in the mid- and far-infrared in case

$\frac{Q_{\mathrm{IR}}}{R_{\text {out }}^{2}}<1.1 \times 10^{-36} \mathrm{~cm}^{-2}$.

For a typical value of $Q_{\mathrm{IR}}$ in the mid- and far-infrared of 0.0028 we find that for $R_{\text {out }}>5.0 \times 10^{16} \mathrm{~cm}$, or $3.4 \times 10^{3} \mathrm{AU}$, the dusty torus is optically thin at these wavelengths. This size is not unreasonable for a circumstellar torus, as it is comparable to the sizes of the dust shell shells around AGB stars, the PN progenitors. In addition, we can argue that the density distribution will not be flat, but probably behaves more like a power law. Then, most lines-of-sight through the torus will be optically thin even in case of a smaller torus. Therefore, we are confident that the torus is indeed optically thin in the mid- and far-infrared.

ii) We assumed that all grains have a size of $0.1 \mu \mathrm{m}$. In reality, the grains formed in the circumstellar environment will cover a wide range of sizes. However, we believe that we actually get a good estimate of the actual dust masses, because we use the mass absorption coefficients, and is thus independent from grain size. Using the mass absorption coefficients will lead to a direct determination of the dust mass, without calculating the mass of individual grains. The calcite and dolomite data presented in this work are mass absorption coefficients $\kappa_{v}$, the data used for diopside, enstatite and forsterite are measured in terms of absorption efficiency $Q_{v} / a$. For amorphous olivine, metallic iron and water ice we used optical constants, and derived absorption efficiency for grains with a size of $0.1 \mu \mathrm{m}$. Only the mass determination of these three species is probably somewhat affected by our assumption on the grain size.

iii) We assumed that all grains are spherical. Again an assumption that is probably not very realistic. The dominant dust component (amorphous olivine) must be in the form of nonspherical particles to explain the infrared-spectrum of AGB stars, and the same is true for metallic iron (Kemper et al. 2002a). The similarity in the spectral appearance of the solid state features detected in PNe and their direct progenitors, AGB stars, (e.g. Sylvester et al. 1999; Molster et al. 2002b), indicates that the dust grains in the two types of objects probably have similar properties. Therefore, we may conclude that the grains in NGC 6302 are very likely not homogeneous spheres, as these particles result in a shift in peak position with respect to nonspherical particles and inhomogeneous spheres. However, in our model the only purpose of the grain shape is to calculate the total mass of some of the dust components. The effect that grain shape has on light scattering is not taken into account, as our model uses mass absorption coefficients.

iv) We assumed that all grains are of homogeneous composition. Using mass absorption coefficients to calculate the emerging spectrum, we simply derived the total mass of a certain mineral found in the line of sight. It is impossible to discriminate between homogeneous grains, and grains consisting of two or more mineral species, therefore we cannot exclude the presence of grains of mixed composition. For instance, it is well known that volatiles, such as water vapour, condense in layered structures on grains (Jura \& Morris 1985). We stress 
that the derived total masses are independent from the assumed homogeneity of grains.

v) We assumed that the density and temperature gradients are simple power laws of the distance to the central star. The complex structure of NGC 6302 indicates that this is probably far besides the truth, but since the optical depth is low, all infrared radiation emitted by the grains is received. Therefore not the density and temperature as a function of distance to the central star, but the mass-temperature relation of the different dust species becomes important, in a similar fashion as described by Bouwman et al. (2000). Our simulation of this relation by a power law density and temperature gradient seems to be in agreement with the observed spectrum.

vi) Finally we assumed that all dust species are found in the same temperature range. However, if the different dust components are not in thermal contact with each other, i.e. if they are present in separate - but co-spatial - grain populations, the difference in optical properties, notably in the UV and visual, causes a difference in the temperature profile (Bouwman et al. 2000; Kemper et al. 2001). The determination of the temperature range is based on the overall shape of the SED, and we forced the crystalline components - which are the carriers of the narrow features superposed on the broad continuum - to have the same temperature profile as the amorphous component. Since the dust emission strongly depends on the temperature of the grains, this will probably be our main source of error in our mass estimates. However, dropping the constraint that all dust species are found in the same temperature range, leads to a large increase in free parameters for our simple model.

Considering all the effects discussed here, we estimate that our absolute mass determinations are accurate within a factor of two, if the distance toward NGC 6302 is 910 pc. If we take into account the large uncertainty in the distance determinations, the error in the absolute dust mass determination further increases. The uncertainty in the various laboratory measurements introduces an additional uncertainty of a factor of two, leading to a total error of a factor of $\sim 3$. This inaccuracy in the laboratory measurements can be inferred from comparison between published data. The crystalline silicate features published by Koike et al. (1999) are intrinsically much stronger (up to 5 times), than the same features in measurements by other groups (Servoin \& Piriou 1973; Steyer 1974; Jäger et al. 1998). Consequently, the crystallinity, defined by the mass of enstatite and forsterite as a fraction of the total dust mass, lies within 5\%-25\%, and the values given in Table 2 are thus just lower limits. The error in the relative dust mass fractions, i.e. the mass ratios between the identified minerals, is much better, as they all depend in the same way on our model assumptions. We therefore estimate the error in the relative dust mass fractions of the order of $50 \%$. The error in the absolute and relative flux levels is small compared to the inaccuracies discussed here, and is therefore not taken into account.

In order to further approach reality, full radiative transfer calculations should be performed. Using spatial information derived from imaging and measurements of outflow velocities it is possible to reconstruct the geometry of the nebula. With 2-dimensional full radiative transfer calculations over a large wavelength range, from UV to far-infrared, it is possible to resolve the temperature and density distribution throughout the PN, for the different dust components. Also, the effect of grain sizes, grain shapes and mineralogical homogeneity then becomes apparent in the spectroscopy. This will lead to a much more accurate description of the properties of the dust in the nebula. However, we chose not to do these calculations for the following reasons: First, optical constants are not available for some of the identified dust components. Second, our knowledge about the geometry of the nebula is still too limited to justify the huge effort of 2-dimensional radiative transfer calculations. These calculations introduce new free parameters, and it will be very hard to constrain the model parameters in an unambiguous way with the limited information available. Spatially resolved spectroscopy of the mid- and far-infrared regions will certainly improve the image we have of NGC 6302. Finally, to obtain reliable mass estimates the distance toward NGC 6302 should be more accurately known.

\subsection{The astronomical relevance of carbonates}

In Paper I and this work, we present the first extrasolar detection of carbonates. The detection is based on far-infrared features of carbonate at $92 \mu \mathrm{m}$ and dolomite, which contributes to the $60 \mu \mathrm{m}$ complex. Due to the low abundances, the strong resonances present at short wavelengths (see Fig. 6) do not stand out in the spectra, as many other species contribute significantly to the opacity at these wavelengths. This is not the case at the far-infrared wavelengths, therefore it is possible to detect even very small amounts of carbonates, beyond reasonable doubt.

There have been a number of searches for carbonate features in the mid-infrared. The previously unidentified infrared (UIR) feature at $11.3 \mu \mathrm{m}$ seen in planetary nebula NGC 7027 was attributed to carbonates (Gillett et al. 1973; Bregman \& Rank 1975). However, this identification became unlikely with the non-detection of the $6.8 \mu \mathrm{m}$ carbonate feature in NGC 7027 (Russell et al. 1977b), and the lack of characteristic features due to carbonates in the $22-35 \mu \mathrm{m}$ region of the spectrum (McCarthy et al. 1978). The UIR feature at $11.3 \mu \mathrm{m}$ in HD 44179, also known as the Red Rectangle, was attributed to the carbonate magnesite $\left(\mathrm{MgCO}_{3}\right)$ (Bregman 1977). However, Cohen et al. (1986) showed PAHs are the most likely carrier of the UIR feature at $11.3 \mu \mathrm{m}$ seen in various astrophysical environments. Thus, the sole detection of the $11.3 \mu \mathrm{m}$ feature is no longer considered evidence for the presence of carbonates.

Interesting environments to search for carbonates are star forming regions and young stars. Carbonates form easily on the surface of planets when liquid water is present, but other formation mechanisms cannot be excluded (Paper I). Russell et al. (1977a) have determined an upper limit to the carbonate/silicate mass ratio in the interstellar medium toward Orion. They find that the this mass ratio is at most 0.05 , based on the detection limit of the $6.8 \mu \mathrm{m}$ feature. The $6.8 \mu \mathrm{m}$ feature is seen in absorption toward embedded protostars. The resemblance of the spectral appearance of the $6.8 \mu \mathrm{m}$ feature in W33A and in Interplanetary Dust Particles (IDPs) has lead to the conclusion that they have the same carrier, most likely carbonates (Sandford \& Walker 1985; Tomeoka \& Buseck 1986), 
although Tielens et al. (1984) argue that it is due to hydrocarbons. Cox (1989) observes the $6.8 \mu \mathrm{m}$ feature towards protostar AFGL 961 and claims that it is partially due to carbonates along with a contribution of hydrocarbons. Hints of the 11.3 and $13.4 \mu \mathrm{m}$ carbonate features are also claimed to be present. The similarity between the $6.8 \mu \mathrm{m}$ features in IDPs and in interstellar lines-of-sight is reported by Quirico et al. (2000) as well, presumably coming from the same carrier, although carbonates are rejected as a carrier. From the high resolution ISO SWS spectroscopy it becomes clear that the spectral shape of the $6.8 \mu \mathrm{m}$ absorption feature observed towards protostars cannot be explained by carbonates, but a satisfactory alternative is still lacking (Keane et al. 2001). Recently, the $\sim 92 \mu \mathrm{m}$ carbonate feature has been detected towards protostar NGC 1333IRAS 4 (Ceccarelli et al., in prep.).

Although extraterrestrial carbonates are quite often found in meteorites and IDPs, they are actually not easy to detect by means of astronomical observations. The only claim still standing at the moment is the detection of features at 26.5 and $31 \mu \mathrm{m}$ due to dolomite and calcite respectively in the ISO SWS spectrum of Mars (Lellouch et al. 2000). However, the far-infrared features of dolomite and calcite at $\sim 60$ and $\sim 92 \mu \mathrm{m}$ are not seen on Mars (Burgdorf et al. 2000).

As pointed out in Paper I, until now carbonates are believed to be formed through aqueous alteration. In this formation process, carbon dioxide $\left(\mathrm{CO}_{2}\right)$ dissolves in liquid water and forms carbonate ions $\left(\mathrm{CO}_{3}{ }^{2-}\right)$. If cations like $\mathrm{Ca}^{2+}$ or $\mathrm{Mg}^{2+}$ are found in the solution as well, carbonates can be formed as a lake sediment when saturation is reached. The presence of carbonates is seen as evidence of planet formation, as an atmosphere containing carbon dioxide is required, as well as liquid water on the surface of the planet. The connection with planet formation is the justification for the search for carbonates in the circumstellar environment of young stars. Carbonates are ubiquitous in our own solar system, as is shown from the composition studies of meteorites and interplanetary dust particles. Carbonate-containing meteorites are considered tracers of the formation history of the solar system (e.g. Endress et al. 1996; Bischoff 1998). However, the detection of carbonates in PNe suggests that alternative formation mechanisms exist and that the presence of carbonates no longer provides direct evidence for planet formation (Paper I).

\section{Summary}

We have determined the composition and distribution of the dust in the planetary nebula NGC 6302. We found that a warm (100-118 K) and a cool (30-60 K) dust component are present. The cool dust component is located in a circumstellar torus of which the inner part effectively shields the UV/optical and near-infrared radition from the central star. The torus contains amorphous olivine, forsterite, clino-enstatite, water ice, diopside, dolomite and calcite. Outside the solar system, the carbonates dolomite and calcite are only seen in NGC 6302 and NGC 6537 (see also Paper I), and the formation mechanism of carbonates in these environments is not yet understood. Diopside is so far only found in NGC 6302 (Koike et al. 2000) and in two OH/IR stars (Demyk et al. 2000). Water ice, forsterite, enstatite and amorphous olivine are very common in the dust shells of evolved stars (e.g. Molster et al. 2002a, b, c). The circumstellar torus also contains the $\mathrm{OH}$ maser reported by Payne et al. (1988).

The optical depth in the polar regions is smaller, and therefore, these regions can be observed in the optical in scattered light from the central star. These regions contain the warm dust component, which thermally emits in the mid-infrared. The $N$-band images are dominated by thermal emission from the warm dust. From the ISO spectroscopy, constraints on the dust composition could be derived: predominantly metallic iron (or another featureless dust component), amorphous olivine, forsterite and clino-enstatite are detected. For water ice, diopside, calcite and dolomite we have derived upper limits for the mass fraction in the warm component. Only $0.3 \%$ of the total dust mass is contained in the warm component, which has a different mineralogical composition than the cool component. UV radiation can penetrate the polar regions and thus we are able to explain the observed PAH emission, which is also reported by Casassus et al. (2000).

In addition, the mass absorption coefficients of carbonate minerals are presented in this work. We studied calcite, dolomite, ankerite, aragonite siderite and magnesite.

Acknowledgements. We thank M. Matsuura for her help in obtaining the TIMMI2 observations and A. de Koter for the useful discussions. We are grateful for the support by the staff of the ESO $3.6 \mathrm{~m}$ telescope. LBFMW and FK acknowledge financial support from an NWO "Pionier" grant. This work was partly supported by NWO Spinoza grant 08-0 to E. P. J. van den Heuvel.

\section{References}

Barlow, M. J. 1998, Ap\&SS, 255, 315

Bertie, J. E., Labbé, H. J., \& Whalley, E. 1969, J. Chem. Phys., 50, 4501

Bischoff, A. 1998, Meteoritics and Planetary Science, 33, 1113

Bohigas, J. 1994, A\&A, 288, 617

Bouwman, J., de Koter, A., van den Ancker, M. E., \& Waters, L. B. F. M. 2000, A\&A, 360, 213

Brearley, A. J., \& Jones, R. H. 1998, in Rev. in Mineralogy, Planetary Materials, ed. J. Papike (Mineralogical Society of America), vol. 36,1

Bregman, J. D. 1977, PASP, 89, 335

Bregman, J. D., \& Rank, D. M. 1975, ApJ, 195, L125

Burgdorf, M. J., Encrenaz, T., Brucato, J. R., et al. 2000, in ISO beyond the peaks: The 2nd ISO workshop on analytical spectroscopy, ESA-SP 456, 9

Casassus, S., Roche, P. F., \& Barlow, M. J. 2000, MNRAS, 314, 657

Cohen, M., Allamandola, L., Tielens, A. G. G. M., et al. 1986, ApJ, 302, 737

Cox, P. 1989, A\&A, 225, L1

Demyk, K., Dartois, E., Wiesemeyer, H., Jones, A. P., \& d'Hendecourt, L. 2000, A\&A, 364, 170

Dorschner, J., Begemann, B., Henning, T., Jäger, C., \& Mutschke, H. 1995, A\&A, 300, 503

Endress, M., Zinner, E., \& Bischoff, A. 1996, Nature, 379, 701

ESO. 1998, ESO Press Release 06/98

Gillett, F. C., Forrest, W. J., \& Merrill, K. M. 1973, ApJ, 183, 87

Gómez, Y., Moran, J. M., Rodríguez, L. F., \& Garay, G. 1989, ApJ, 345,862 
Gómez, Y., Rodríguez, L. F., \& Moran, J. M. 1993, ApJ, 416, 620

González-Alfonso, E., \& Cernicharo, J. 1999, ApJ, 525, 845

Hellwege, K. H., Lesch, W., Plihal, M., \& Schaack, G. 1970, Z. Physik, 232, 61

Henning, T., Il'In, V. B., Krivova, N. A., Michel, B., \& Voshchinnikov, N. V. 1999, A\&AS, 136, 405

Henning, T., \& Stognienko, R. 1996, A\&A, 311, 291

Hoogzaad, S. N., Molster, F. J., Dominik, C., et al. 2002, A\&A, 389, 547

Jäger, C., Molster, F. J., Dorschner, J., et al. 1998, A\&A, 339, 904

Johnson, C. A., \& Prinz, M. 1993, Geochim. Cosmochim. Acta, 57, 2843

Jura, M., \& Morris, M. 1985, ApJ, 292, 487

Keane, J. V., Tielens, A. G. G. M., Boogert, A. C. A., Schutte, W. A., \& Whittet, D. C. B. 2001, A\&A, 376, 254

Kemper, F., de Koter, A., Waters, L. B. F. M., Bouwman, J., \& Tielens, A. G. G. M. 2002a, A\&A, 384, 585

Kemper, F., Jäger, C., Waters, L. B. F. M., et al. 2002b, Nature, 415, 295

Kemper, F., Waters, L. B. F. M., de Koter, A., \& Tielens, A. G. G. M. 2001, A\&A, 369, 132

Koike, C., Tsuchiyama, A., Shibai, H., et al. 2000, A\&A, 363, 1115

Koike, C., Tsuchiyama, A., \& Suto, H. 1999, in Proc. 32nd ISAS Lunar Planet. Symp., 175

Lellouch, E., Encrenaz, T., de Graauw, T., et al. 2000, Planet. Space Sci., 48, 1393

Lester, D. F., \& Dinerstein, H. L. 1984, ApJ, 281, L67

McCarthy, J. F., Forrest, W. J., \& Houck, J. R. 1978, ApJ, 224, 109

Molster, F. J., Lim, T. L., Sylvester, R. J., et al. 2001a, A\&A, 372, 165

Molster, F. J., Waters, L. B. F. M., \& Tielens, A. G. G. M. 2002a, A\&A, 382, 222

Molster, F. J., Waters, L. B. F. M., Tielens, A. G. G. M., \& Barlow, M. J. 2002b, A\&A, 382, 184
Molster, F. J., Waters, L. B. F. M., Tielens, A. G. G. M., Koike, C., \& Chihara, H. 2002c, A\&A, 382, 241

Molster, F. J., Yamamura, I., Waters, L. B. F. M., et al. 2001b, A\&A, 366, 923

Orofino, V., Blanco, A., Fonti, S., Proce, R., \& Rotundi, A. 1997, Planet. Space Sci., 46, 1659

Payne, H. E., Phillips, J. A., \& Terzian, Y. 1988, ApJ, 326, 368

Penman, J. M. 1976, MNRAS, 176, 539

Persi, P., Cesarsky, D., Marenzi, A. R., et al. 1999, A\&A, 351, 201

Pottasch, S. R., Beintema, D., Dominguez-Rodriguez, F. J., et al. 1996, A\&A, 315, L261

Pottasch, S. R., \& Beintema, D. A. 1999, A\&A, 347, 975

Quirico, E., Raynal, P.-I., Borg, J., et al. 2000, in Lunar and Planetary Sci. Conf., 31, 1260

Reimann, H.-G., Linz, H., Wagner, R., et al. 2000, Proc. SPIE, 4008, 1132

Roche, P. F., \& Aitken, D. K. 1986, MNRAS, 221, 63

Russell, R. W., Soifer, B. T., \& Puetter, R. C. 1977a, A\&A, 54, 959

Russell, R. W., Soifer, B. T., \& Willner, S. P. 1977b, ApJ, 217, L149

Sandford, S. A., \& Walker, R. M. 1985, ApJ, 291, 838

Schwarz, H. E., Corradi, R. L. M., \& Melnick, J. 1992, A\&AS, 96, 23

Servoin, J. L., \& Piriou, B. 1973, Phys. Stat. Sol., 55, 677

Shaw, R. A., \& Kaler, J. B. 1989, ApJS, 69, 495

Snow, T. P. 1984, ApJ, 287, 238

Steyer, T. R. 1974, Ph.D. Thesis, University of Arizona, USA

Sylvester, R. J., Kemper, F., Barlow, M. J., et al. 1999, A\&A, 352, 587

Tielens, A. G. G. M., Allamandola, L. J., Bregman, J., Goebel, J., \& d'Hendecourt, L. B. 1984, ApJ, 287, 697

Tomeoka, K., \& Buseck, P. R. 1986, Science, 231, 1544

Warren, S. G. 1984, App. Opt., 23, 1206

Yamagishi, K., Saeki, T., Koike, C., \& Chihara, H. 2001, Meteoritics and Planetary Science, 36, 228 